\title{
Design, construction and performance of magnetised minilCAL detector module
}

Gobinda Majumder*, E-mail: gobindaetifr.res.in,

Tata Institute of Fundamental Research, Mumbai,

Suryanaraya Mondal ${ }^{\dagger}$ E-mail: suryanarayan.mondaletifr.res.in

Tata Institute of Fundamental Research, Mumbai \& Homi Bhabha National Institute, Mumbai

(On behalf of the miniICAL group)

India-based Neutrino Observatory (INO) has proposed to build a 51 kton magnetised Iron Calorimeter (ICAL) in an underground laboratory to be constructed near Madurai, South India. ICAL is aimed to study the atmospheric neutrino oscillation parameters precisely and determine the ordering of neutrino masses. ICAL will be built by stacking 151 layers of $56 \mathrm{~mm}$ soft iron plates, spanning essentially a lateral area of $48 \mathrm{~m} \times 16 \mathrm{~m}$. The detector will deploy about 28,800 glass Resistive Plate Chambers (RPCs) of approximately $2 \mathrm{~m} \times 2 \mathrm{~m}$ in area, inserted between the iron layers. About 3.6 million detector channels are required to be instrumented to read data.

During the detector R\&D phase, many RPC detector stacks were constructed and operated by the collaboration for prolonged periods of time - several years, mainly studying long term performance of the RPC detectors. But a magnetised 'miniICAL' was proposed to be built, essentially exactly of the same design as that of ICAL and also using same detector components. This was expected to serve the purpose of understanding the engineering issues in constructing the ICAL, and at the same time provide important inputs on the ICAL's operating parameters and physics measurement capabilities. A veto wall is also being planned around the miniICAL, to explore possibility of building ICAL like detectors on surface or at shallow depths. The veto wall will be made of extruded scintillator tiles and coupled to SiPMs through WLS fibres.

The experiment was built using 11 layers of $56 \mathrm{~mm}$ soft iron plates, spanning a lateral area of $4 \mathrm{~m} \times 4 \mathrm{~m}$. A nominal magnetic field of 1.5 Tesla is produced inside the iron plates, by passing about 900 A current through two, 18-turn OFHC copper coils which are water-cooled. A total of 10 RPCs of about $1.9 \mathrm{~m} \times 1.9 \mathrm{~m}$ in area is inserted between the iron layers and readout using the electronics of the same design as that of ICAL.

The 39th International Conference on High Energy Physics (ICHEP2018)

4-11 July, 2018

Seoul, Korea

*Speaker.
${ }^{\dagger}$ Corresponding author

(C) Copyright owned by the author(s) under the terms of the Creative Commons 


\section{Introduction}

The long term stability of resistive plate chamber (RPC) detector was tested using 12 RPC stack of $1 \mathrm{~m} \times \mathrm{m}$ at Mumbai as well as 12 RPC stack of $2 \mathrm{~m} \times 2 \mathrm{~m}$ RPC stack at Madurai. This test was down with different electronics including the final design for the INO-ICAL experiment [1]. But, those were tested in absence of magnetic field. To validate all those electronics in the fringe field of ICAL magnet, the miniICAL detector is constructed. Out of 20 layers of RPC, 10 layers are operational now. Other than the test of electronics in presence of magnetic field, these following items are being tested in this setup (i) magnetic field measurement using pickup coil and Hall probes and compare with 2D simulation by MagNet7, (ii) performance of RPC including DC-DC power supply, (iii) feasibility study of using Muon Spin Rotation to measure B-field, complementary to sense wire loop and Hall probe data, (iv) measurement of the charge dependent muon flux upto $\sim 1.5 \mathrm{GeV}$ and compare with simulation, $(v)$ proof of principle test of the cosmic muon veto detector for the feasibility of a shallow ICAL detector etc.

\section{Detector}

The sensitive detector are this miniICAL is RPCs. Each RPC has two readout planes, either side of the gas gap, labeled as X-and Y-planes. Each plane has 60/63 strips of width $2.8 \mathrm{~cm}$ with an inter-strip gap of $0.2 \mathrm{~cm}$. The readout planes are arranged with pickup strips oriented orthogonal to each other so that they can record a 2D position information of the traversing charged particle.

This detector is $\sim 0.1 \%$ of the final ICAL detector. electronics. A view of the detector is shown in figure 1 . The major components of this detector are described briefly here. The 85 ton electromagnet of low carbon iron in 11 layers, the spacers maintaining the required gaps for insertion of the RPCs, the magnetic field measurement system, a DC power supply (PS) and the copper coils for energizing the magnet. The RPC based muon detectors including (i) $1.9 \times 1.9 \mathrm{~m} \times 8 \mathrm{~mm}$ glass gap, (ii) front-end electronics which includes 128 channels of fast amplifier and discriminator, (iii) digital back-end (DFE) electronics which comprises of several functional blocks, e.g., a TDC, strip-hit latch, rate monitor, pre-trigger generator, ambient parameter monitor and Front-end control, (iv) a DC-DC high voltage supply based on a current fed resonant Royer circuit, $(v)$ a data acquisition board, (vi) low voltage supply lines and gas lines packed in a steel reinforced epoxy tray. Other electronics, consists of multilevel trigger system which includes logic, essentially defined as $m \times p / n$, i.e., trigger is generated when out of a group of $n$ consequent layers, at least $p$ layers have $m$ channels each with simultaneous signals in them, calibration unit mainly interfaces with trigger system and DFE boards and performs functions of distribution of global clocks and trigger signals as well as measurement of time offsets due to disparate signal path lengths and pulse shaper. A closed loop gas system including gas purified to circulate the mixture of R134a, Isobutane and $\mathrm{SF}_{6}$ with a proportion of 95.2:4.5:0.3. Flow rate is $6 \mathrm{SCCM}$ per chamber and maintained 2-3 mbar over pressure with respect to the atmospheric pressure.A closed loop low conductivity ( $\leq 10 \mu \mathrm{mho} / \mathrm{cm}$ ) chilled water system to cool the magnet power supply system and the conducting copper coils. Slow controls and monitoring of gas, magnet, power supply, ambient parameters, like pressure, temp, humidity etc. Back-end data concentrator, event builder, data storage system. 

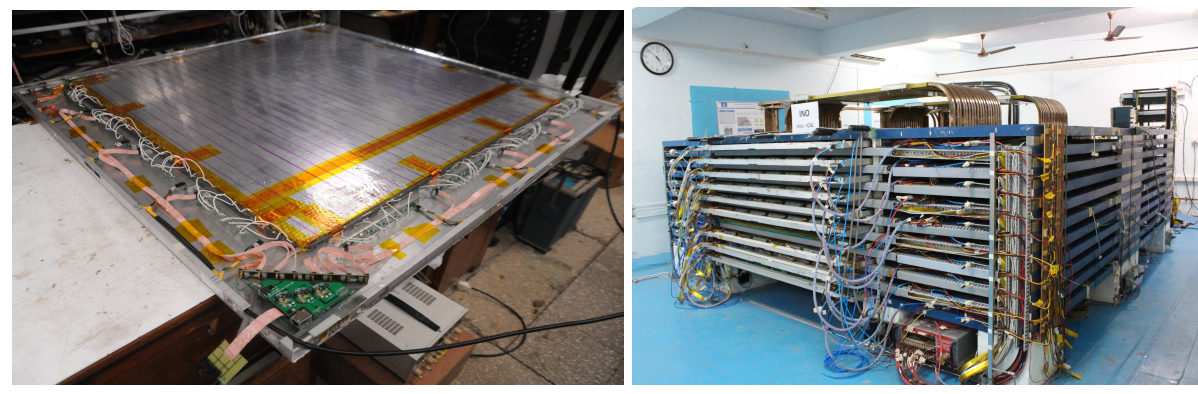

Figure 1: (left) A RPC with complete readout electronics and (right) A view of miniICAL detector with 10 layers of RPC detector.

\section{Comparison of signal with and without magnetic field}

According to the design, the magnetic field is maximum and also nearly uniform inside the coil. The expected magnetic field in different position in layer-3 is shown in figure 2 . There are about 150 Hall probes to measure magnetic field in different position in a layer.

Measured field in different position are within $10 \%$ of the expected field from the the simulation. According to the ratio of the field in simulation and observed data, simulated magnetic field map $(5 \mathrm{~cm} \times 5 \mathrm{~cm}$ grid points) are modified. During event simulation and reconstruction of $\mathrm{MC}$ and real data, magnetic field at any position is calculated using simple interpolation technique.
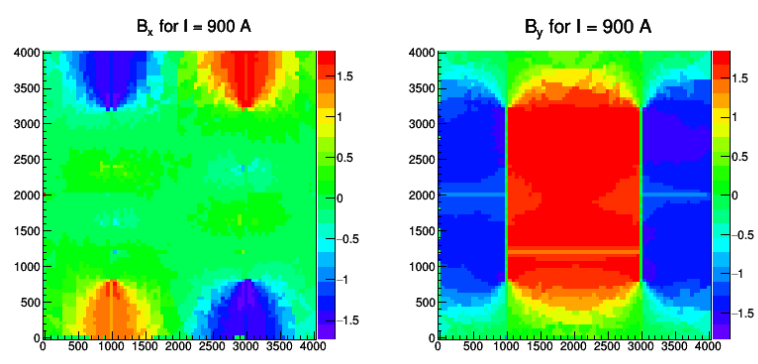

Figure 2: Magnetic field map at $900 \mathrm{~A}$ current, (left) $\mathrm{B}_{x}$ and (right) $\mathrm{B}_{y}$ in Tesla. Length in $\mathrm{X}-\& \mathrm{Y}$ - axis are in $\mathrm{mm}$.

Depending on the gain of the RPC, the strip noise varies from about $50 \mathrm{~Hz}$ to $500 \mathrm{~Hz}$. Strip noise as a coil current is shown in figure 3 for few strips, where current in the coil reduces smoothly from $900 \mathrm{~A}$ to zero. It is absolutely clear that there is no change in noise rate with the magnetic field. There is no effect of magnetic field on pulse shape of the signal as well as measurement of timing.

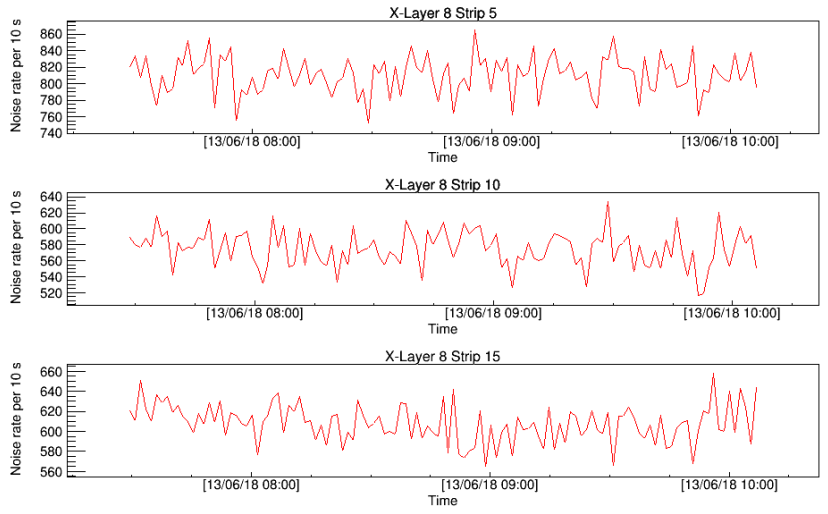

Figure 3: Noise rate in RPC strips for continuous decrease of coil current from 990 A to 0. 
Some typical events, at 900A current, corresponds to magnetic field at $\sim 1.5 \mathrm{~T}$ in the central region is shown in figure 4 . In the central region, magnetic field is almost parallel to $\mathrm{Y}-$ axis, thus the bending of muon trajectory in $\mathrm{X}-\mathrm{Z}$

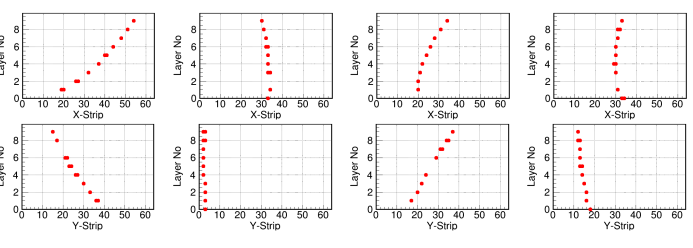
plane. Apparent bending in Y-Z plane is due to Figure 4: Typical cosmic muon events in minilarge multiple scattering of muon in iron plate. ICAL; (top) X-Z view and (bottom) Y-Z view.

\section{Cosmic muon spectrum at Madurai}

The ICAL reconstruction code [2] is used to reconstruct tracks, except the change in geometry and magnetic field. Normalised momentum, polar angle and azimuthal angle distribution of data and MC sample are shown in figure 5. Due to limited number of layer and poorer position resolution $\left(\sigma_{x, y} \sim 7-8 \mathrm{~mm}\right)$ with respect to conventional tracking device $\left(\sigma_{x, y} \leq 100 \mu \mathrm{m}\right)$, the momentum resolution is poor. Due to comparatively poor momentum resolution this spectrum need to unfold to get the true muon spectrum at Madurai to compare the muon predicted momentum spectrum at Madurai.
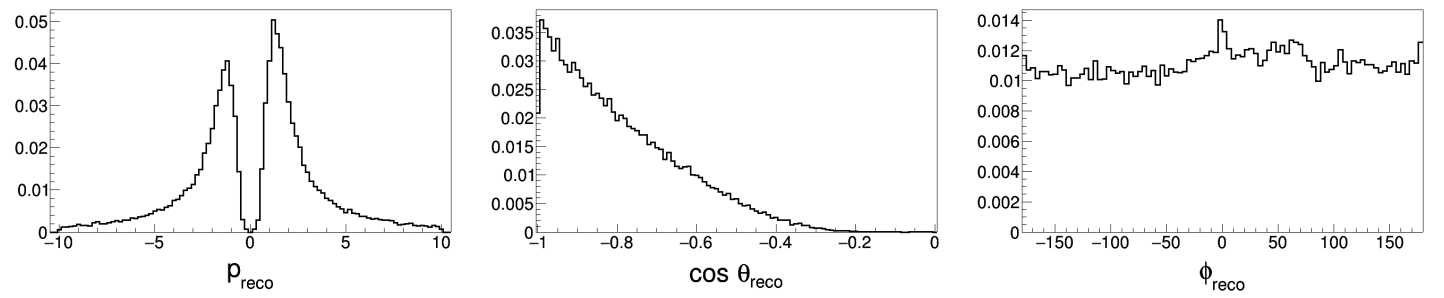

Figure 5: Reconstructed muon momentum (-ve value implies $\mu^{-}$), cosine of zenith angle and azimuthal angle of cosmic muon recorded in miniICAL.

\section{Conclusion}

Out of 20 RPC gaps, 10 RPC gaps are commissioned in 83 ton miniICAL detector and remaining 10 will be installed soon. There is no visible effect magnetic field on the performance of RPC and related electronics. This detector is continuously accumulating large muon sample to measure muon kinematics at Madurai, which may improve the precision of the prediction of neutrino flux at INO site. Muon spin rotation technique to measure magnetic field using this setup is required better algorithm to improve signal to background ratio on top layer. Cosmic Muon Veto detector with three layers of extruded scintillator is going to integrate with this system soon.

\section{References}

[1] Ahmed, Shakeel and others, Physics Potential of the ICAL detector at the India-based Neutrino Observatory (INO), Pramana 88 (2017) no.5, 79.

[2] S. Seth, A. Bhatt, G. Majumder and A. Mishra, Update of INO-ICAL reconstruction algorithm, JINST 13 (2018) no.09, P09015 